\title{
COVID-19 y salud reproductiva
}

\section{COVID-19 and reproductive health}

\author{
Gómez-Tabares $G^{1}$,Barraza-Gerardino $M^{2}$
}

${ }^{1}$ Ginecología y Obstetricia. Endocrinología Reproductiva y Fertilidad. Centro Médico Imbanaco, Cali, Colombia. Profesor titular y distinguido, Universidad del Valle. Profesor, Hora Catedra, Universidad del Valle; Universidad Libre, Cali, Colombia.

${ }^{2}$ Medicina Interna, Endocrinología, Colsánitas, IPS Vivir Cali, Cali, Colombia.

Autor de correspondencia: Mónica Barraza Gerardino Correo electrónico: gustavo.gomez@imbanaco.com.co

\section{Resumen}

El comportamiento de las enfermedades microbianas, ya sea por virus, bacterias o protozoos, y su respuesta inflamatoria son diferentes entre hombres y mujeres. Esta diferencia se hace notoria en la pandemia derivada por la enfermedad por coronavirus (COVID-19). Desde el reporte del primer caso de neumonía en diciembre de 2019, en Wuhan, China, la COVID-19 se ha diseminado a 212 países y territorios y, a la fecha, se ha confirmado más de 3,5 millones de casos, con una mortalidad mundial del 7\%, lo que la convierte en una emergencia sanitaria internacional ${ }^{(1)}$. Hasta ahora, en Colombia, hay más de 7000 casos confirmados, con más de 300 defunciones, de los cuales, más del $60 \%$ pertenecen al sexo masculino. Hasta el momento, la literatura científica disponible relacionada con la COVID-19 solo abarca ciertos aspectos de la salud reproductiva, tanto femenina como masculina, mientras se continúa recopilando más información que nos permita conocer y realizar un análisis más detallado de su impacto real en humanos durante el proceso infeccioso y las secuelas derivadas de este. Está confirmado que las condiciones médicas relacionadas con el síndrome metabólico y los estados de insulinorresistencia en hombres y mujeres agravan la presentación clínica y el pronóstico ${ }^{(2)}$. La presente revisión pretende ilustrar los mecanismos relacionados con la respuesta inmunitaria diversa frente a las infecciones virales según el sexo del individuo, su compromiso gonadal y los efectos relacionados con la salud reproductiva masculina y femenina, que incluye la maternofetal y la posible transmisión vertical.

Palabras clave: COVID-19, gónadas, función gonadal masculina, hormonas sexuales, sistema reproductivo.

\section{Abstract}

The microbial diseases behavior by viruses, bacteria or protozoa and inflammatory response show differences between men and women. This difference has become noticeable in the face of the emerging from Coronavirus Disease (COVID-19). Since the first case of pneumonia in December 2019 in Wuhan-China, it has been spread to 212 countries and territories, and more than 3.5 million cases have been confirmed with a whole mortality of $7 \%$, making it an global health emergency ${ }^{(1)}$. In Colombia there are until now, more than 7000 confirmed cases with more than three hundred deaths, of which more than $60 \%$ are male. To date, the available scientific literature related to COVID-19 only covers certain aspects of reproductive health, both female and male, as more information is gathered that allows us to know and carries out a more detailed analysis of its real impact on humans during the infectious process and the sequelae. Medical conditions related to metabolic syndrome and insulin resistance in men and women aggravate the clinical presentation and prognosis ${ }^{(2)}$. This review aims to illustrate the mechanisms related to the diverse immune response to viral infections according to the individual's sex, their gonadal involvement, and effects related to male and female reproductive health, including maternal fetal health and possible vertical transmission.

Keywords: COVID-19, Gonads, Vertical transmission, Pregnancy, Male gonadal function, Sex-related hormones, Reproductive system.

\section{Introducción}

La enfermedad por coronavirus 2019 (COVID-19) es una infección respiratoria aguda potencialmente grave, causada por el coronavirus de la misma especie del responsable del síndrome respiratorio agudo grave (SARS-CoV-2), que se identificó como el causal del brote de neumonía de origen previamente desconocido en 44 pacientes en la ciudad de Wuhan, provincia de Hubei, China, el 31 de diciembre de $2019^{\text {(3). Los }}$ coronavirus son una familia numerosa de virus del ácido ribonucleico (ARN), de los cuales, algunos causan enfermedades en humanos, como el resfriado común, el síndrome respira- 
torio agudo grave (SARS), el síndrome respiratorio de Oriente Medio [MERS] y otros que se propagan entre mamíferos y aves. Con escasa frecuencia, los coronavirus de animales pueden transmitirse a los humanos y entre humanos, como fue el caso del SARS y el MERS ${ }^{(4,5)}$. El SARS-CoV-2 pertenece al subgénero de Sarbecovirus de la familia coronaviridae y es el séptimo coronavirus del cual se conoce que infecta a seres humanos. Este virus presenta características similares a las del coronavirus del SARS proveniente de los murciélagos ${ }^{(6,7)}$. Un estudio preliminar sugiere que hay dos cepas principales del virus del SARS-CoV-2 en China, designados L y S. Se determinó que el tipo L era más prevalente durante las primeras etapas del brote en la ciudad de Wuhan y se especula que podría ser el más agresivo ${ }^{(8)}$.

Los datos de la mayor serie de casos de China revelan que el $87 \%$ de los casos confirmados tenía entre 30 y 79 años, el $1 \%$ tenía 9 años o menos, el 1\% tenía entre 10 y 19 años y el $3 \%$ tenía 80 años o más. Aproximadamente, el 51\% de los pacientes eran hombres. Cerca del $4 \%$ de los casos correspondía profesionales de la salud ${ }^{(9)}$.

De acuerdo con los reportes actuales, la COVID-19 tiene un curso más agresivo en el sexo masculino. El $82 \%$ de los pacientes que ingresaron a la unidad de cuidados intensivos (UCI) por falla respiratoria hipoxémica por COVID-19 eran hombres, según un estudio italiano retrospectivo realizado en 72 hospitales. La distribución fue similar por edades en todos los grupos ${ }^{(10)}$. En Colombia, más del $60 \%$ de los fallecidos han sido de sexo masculino y ocupan el $60 \%$ de la estancia en UCI ${ }^{(11)}$. En China, la tasa de mortalidad es $48 \%$ mayor en hombres que en mujeres y se ha observado que, en mujeres gestantes, el compromiso es menos agresivo ${ }^{(12)}$. Con base en esta diferencia, se ha explorado los variados mecanismos que expliquen la mayor agresividad de la enfermedad en hombres y una condición aparentemente protectora en mujeres, incluidos las gestantes y los niños. La susceptibilidad ante procesos infecciosos virales está relacionada con la respuesta innata, adaptativa y celular, en la que los esteroides sexuales cumplen una importante labor en su mediación ${ }^{(13)}$ con receptores de células diana, vías de señalización celular y expresión de factores relativos a los cromosomas sexuales. A continuación, se revisarán los reportes relativos al comportamiento viral según el sexo en gestantes y los mecanismos que explican la diferencia en la respuesta inmunitaria viral.

La información fue obtenida de los motores de búsqueda PubMed, Embase y Google Académico utilizando los siguientes términos: "Covid-19", "hormonas sexuales", "gónadas" y "embarazo". Se revisó un total 56 de artículos que incluye manuscritos aprobados para publicación.

\section{Dinámica de la transmisión}

La evaluación inicial sobre los primeros 425 casos confirmados reveló que el 55\% de los casos anteriores al 1 de enero de 2020 estaba vinculado al mercado de mariscos de Huanan, en el sur de China, mientras que solo el $8,6 \%$ de los casos posteriores a dicha fecha presentaba algún vínculo con este mercado, lo cual confirma que la transmisión entre seres humanos se produjo entre contactos cercanos a partir de mediados de diciembre de 2019, lo que incluía las infecciones entre profesionales sanitarios ${ }^{(6)}$. Cada vez se reconoce más la transmisión en cadenas de varios eslabones a través de un estrecho contacto con las gotas producidas después de la exhalación, los estornudo o la tos, el contacto directo o a través de un vector pasivo, que puede ser cualquier objeto inanimado que logre ingresar a las mucosas respiratorias ${ }^{(14)}$. Aún no es claro cuáles son las otras vías de transmisión, pero se ha encontrado partículas virales en otros fluidos corporales, como la sangre, el líquido cefalorraquídeo, la saliva, las lágrimas, las secreciones conjuntivales y casi la mitad de las muestras de heces de los pacientes ${ }^{(15-17)}$. La detección en el líquido peritoneal y en el semen ha alertado de otros mecanismos de contagio ${ }^{(18,19)}$. Es posible que también sean determinantes la carga viral y el estado de inmunocompetencia del huésped para adquirir la enfermedad.

La transmisión presintomática parece estar relacionada con gestantes y niños afectados por la COVID-19. De los escasos estudios disponibles, se sugiere que algunas personas pueden ser contagiosas durante el período de incubación, que está estimado entre 1 y 14 días, con una mediana de 5 a 7 días (posiblemente más largo en los niños). Aproximadamente, el 97,5\% de los pacientes desarrollan síntomas en los primeros 11,5 días de contraída la infección ${ }^{(20-22)}$. Se ha informado la transmisión presintomática en el $12,6 \%$ de los casos en China ${ }^{(23)}$.

\section{Coronavirus durante el embarazo y transmisión vertical}

Se desconoce si es posible la transmisión perinatal (incluida la transmisión a través de la lactancia materna). Ciertas revisiones retrospectivas en mujeres embarazadas con COVID-19 no evidenciaron infección intrauterina (24-26). Sin embargo, no se puede descartar la transmisión vertical. De los datos de infecciones por otros coronavirus, como el síndrome respiratorio agudo grave (SARS), que afectó a más de 8400 personas en el mundo, se estimó un compromiso aproximado de 100 gestantes, con una tasa de letalidad hasta del 30\%, con peores desenlaces al compararlas con mujeres no gestantes y complicaciones adicionales dadas por abortos espontáneos y restricción del crecimiento intrauterino. Sin embargo, no se reportó transmisión vertical, lo que sugiere que las complicaciones son secundarias al compromiso materno y no a la infección directa del feto ${ }^{(27)}$. Si bien el análisis placentario fue limitado, no se demostró compromiso de las vellosidades coriónicas ni vasos sanguíneos fetales, hallazgo histopatológico típico en infecciones por diseminación hematógena a través de la placenta. Del MERS-CoV reportado en 11 gestantes, tam- 
bién se presentaron muertes maternas y partos prematuros, pero tampoco se documentó transmisión vertical. A diferencia de los agentes previos, se reportaron casos de infección en neonatos nacidos de madres con COVID-19. Uno de ellos, en Wuhan, nació a término, con adecuada adaptación neonatal, cursó con linfopenia y alteración de las pruebas hepáticas e hisopado faríngeo positivo para COVID-19, tomado a las 36 horas posparto, aunque en un hospital que no contaba con las condiciones de aislamiento para el neonato. No obstante, también realizaron las pruebas de ácido nucleico de especímenes placentarios y del cordón umbilical, las cuales fueron negativas, así como en la leche materna. Hasta la fecha, no es clara la posibilidad de transmisión vertical, aunque no se puede descartar debido a varios factores tales como la limitación de los métodos actuales para detectar una carga viral insuficiente ${ }^{(28)}$. Se ha informado la presencia de anticuerpos específicos contra el virus de la COVID-19 en muestras de suero de neonatos (29-31). Los informes de recién nacidos con sufrimiento fetal o que requirieron ingreso a la UCI y de un nacimiento sin vida después de la enfermedad materna por COVID-19 durante el tercer trimestre sugieren la posibilidad de una patología placentaria inducida por el virus.

Hasta el momento, se ha reportado 1 caso de aborto espontáneo durante el segundo trimestre de embarazo en una mujer con COVID-19, en el que no se identificó otra causa de muerte que, a diferencia de publicaciones previas, parece estar relacionada con la infección placentaria por SARS-CoV-2, respaldado por hallazgos virológicos en la placenta, mas no en el feto. Lo autores puntualizan que la infección del lado materno de la placenta que induce insuficiencia placentaria aguda o crónica podría causar aborto espontáneo o restricción del crecimiento fetal, como se observó en el $40 \%$ de las infecciones maternas por coronavirus del síndrome respiratorio del Medio Oriente y coronavirus del síndrome respiratorio agudo grave $^{(32,33)}$. Recientemente se publicó un caso con compromiso neurológico del recién nacido, que según los autores comprobaba la transmisión vertical ${ }^{(34)}$.

En Wuhan, de diciembre de 2019 a marzo de 2020, se identificaron 118 mujeres embrazadas con neumonía por COVID-19, de las cuales, 84 (71\%) fueron positivas para la prueba de reacción en cadena de la polimerasa (PCR) para casos graves de SARS-CoV-2; las 34 restantes (29\%) tuvieron hallazgos sugestivos en la tomografía computarizada (TC) de tórax. En ese momento, las mujeres embarazadas representaban el $0,25 \%$ de los pacientes con SARS-CoV-2, con edad promedio de 31 años (28 a 34); el 52\% eran nulíparas y el 64\% habían sido infectadas por SARS-CoV-2 durante el tercer trimestre. Los síntomas más comunes fueron fiebre (75\%) y tos (73\%); el $44 \%$ presentó linfopenia y el 79\% mostraba infiltrados bilaterales en la TC de tórax. El 8\% de las pacientes desarrolló enfermedad grave y una de ellas requirió ventilación mecánica no invasiva. Después del parto, 6 de las 9 mujeres desarro- llaron enfermedad grave. No hubo muertes. De los 68 partos atendidos durante el estudio, el 93\% fueron por cesárea. No se reportó asfixia neonatal. La prueba para SARS-CoV-2 se realizó en el hisopado faríngeo de 8 recién nacidos y en las muestras de leche materna de 3 madres, cuyos resultados fueron negativos. Los autores concluyeron que su población embarazada (8\%) presentaba un desenlace favorable, de acuerdo con el riesgo reportado en la población general de pacientes con COVID-19 en China continental $(15,7 \%)^{(35)}$. Datos anteriores han mostrado tasas más bajas de enfermedad grave entre mujeres y pacientes jóvenes que entre hombres y pacientes mayores (36). Los datos actuales no sugieren un mayor riesgo de enfermedad grave entre mujeres embarazadas, efecto contrario al observado con el virus de la influenza ${ }^{(37)}$.

Con base en la experiencia del Hospital Presbyterian Allen de Nueva York y el Centro Médico Columbia de la Universidad de Irving ${ }^{(38)}$, entre el 22 de marzo y el 4 de abril de 2020 se atendieron 215 partos. De estas, 4 maternas $(1,9 \%)$ tenían fiebre u otros síntomas de COVID-19 confirmada al ingreso. De las 211 mujeres sin síntomas de COVID-19 se obtuvieron hisopados nasofaríngeos en 210 (99,5\%), de las cuales, 29 (13,7\%) fueron positivas para SARS-CoV-2; de estas, el 87,9\% eran asintomáticas y $3(10 \%)$ desarrollaron fiebre alta antes del posparto, con una mediana de estancia de 2 días. Los autores concluyeron enfatizando en la importancia de realizar pruebas universales de SARS-CoV-2 en todas las pacientes que se presenten para el parto. Ambos reportes sugieren que, al igual que los niños, la menor gravedad de la enfermedad posiblemente está relacionada con una menor expresión de los receptores de la enzima convertidora de la angiotensina (ECA-2) en las mujeres embarazadas, que brinda cierto grado de protección por su estado hormonal o, tal vez, por la influencia del feto en la madre.

\section{COVID-19 e interacción con los receptores}

Para el ingreso del coronavirus a la célula diana, es necesaria la unión de la subunidad S1 de la glucoproteína de la superficie de la espiga viral (S) con los receptores de la ECA-2, al igual que lo hace el virus del SARS-CoV ${ }^{(39,40)}$. Por otro lado, la proteína $\mathrm{S}$ viral es acoplada a una proteína transmembrana con actividad serina proteasa tipo II codificada por el gen TMPRSS2, que está conformada por tres dominios: el dominio proteasa de $\mathrm{S} 1$, que permite la entrada y activación viral, el dominio LDLRA, que se une al calcio, y el dominio transmembrana ${ }^{(41)}$. La actividad del receptor de andrógenos (AR) es requerida para la transcripción del gen TMPRSS2 ${ }^{(42)}$. Este gen también desempeña un papel importante en la patogenia del cáncer de próstata, debido a que cuenta con un elemento de respuesta a la testosterona y dihidrotestosterona en la región promotora. Cuando se fusiona con otros genes de la familia de oncogenes ETS, como el gen ERG, que codifica para una proteína nuclear que actúa como factor de transcripción en áreas ricas en purina, trae como consecuencia cambios en el compor- 
tamiento biológico y la agresividad del cáncer, pero, asimismo, se ha convertido en un blanco terapéutico de esta enfermedad ${ }^{(43)}$. Se ha encontrado expresión de la proteasa TMPRSS2 en espermatogonias y espermátides ${ }^{(44)}$.

Por otro lado, una de las teorías que explica la mayor tasa de morbimortalidad en personas mayores y, especialmente, del sexo masculino es la mayor expresión de la ECA-2, que si bien su presencia se ha descrito en tejido vascular (endotelio, células angiogénicas y músculo liso), corazón, riñones, hígado, retina, sistema nervioso central, vía aérea superior, células epiteliales alveolares tipo II y vasculatura pulmonar ${ }^{(45)}$, también se expresa en las células de Sertoli y Leydig de humanos, mas no en células endoteliales ni germinales, como sí lo hace su homóloga, la ECA. Su presencia sugiere un papel en la regulación de la esteroidogénesis gonadal ${ }^{(46)}$. Por otro lado, la expresión de la ECA parece estar regulada por andrógenos en el miocardio. También se ha descrito en las células de la granulosa ovárica, cuya expresión es estimulada tras el aumento de la hormona luteinizante (LH).

\section{COVID-19, receptores y hormonas sexuales}

Los primeros datos muestran que las mujeres están menos afectadas en número y gravedad por la COVID-19, al igual que las mujeres embarazadas con aparente inmunidad contra el virus, por cursar con sintomatología de menor gravedad y curso más favorable de la enfermedad. Esto ha motivado la investigación del papel del estrógeno y la progesterona en hombres y mujeres con la regulación inmunitaria ante procesos virales. Reportes de infecciones por SARS-CoV y MERS-CoV han demostrado que esta diferencia se va haciendo menos notoria a medida que la edad de la mujer avanza, hasta que pierde esta ventaja en mayores de 70 años ${ }^{(47)}$.

La presencia de dos cromosomas $\mathrm{X}$ fortalece al sistema inmunitario femenino gracias a la interacción sobre varios elementos: sobre los receptores de tipo Toll (Toll Like Receptors - TLR), que son receptores transmembrana tipo 1 expresados por las células presentadoras de antígeno del tejido linfoide y no linfoide ${ }^{(48)}$. En la infección por COVID-19, se produce una sobreexpresión de TLR7 en las células dendríticas pulmonares, los monocitos circulantes, los macrófagos y las células $\mathrm{B}$, que reconocen la cadena de ARN del COVID-19 y, en consecuencia, promueven la producción de anticuerpos anti-COVID-19 y regulan las citocinas proinflamatorias, como la interleucina 6 (IL-6) e IL-1.

$\mathrm{Al}$ activarse, los receptores de estrógenos regulan la inmunidad innata, adaptativa y celular ${ }^{(49)}$, lo que confiere una respuesta más eficiente contra las infecciones virales. Los monocitos, macrófagos y neutrófilos exhiben una mayor expresión de receptores estrogénicos, que inducen la producción de citocinas inflamatorias (IL-12 y factor de necrosis tumoral alfa [FNT- $\alpha]$ ) y quimiocinas (CC2). En los linfocitos inducen la producción de interferón (IFN) tipo I y III. El receptor estrogénico tipo alfa $(\mathrm{ER} \alpha)$ interviene en la regulación y la maduración de las células inmunitarias con un efecto inmunoprotector. De esta manera, permite que las células dendríticas pulmonares, que son la primera línea de defensa en hembras, produzcan más IFN tipo 1 que los machos, mediante la interacción entre los estrógenos y el ER $\alpha^{(13)}$.

En modelos murinos de diferentes edades que se infectaron vía inhalatoria con coronavirus causal del SARS-CoV, se demostraron peores desenlaces en machos y hembras ovariectomizadas o tratadas con tamoxifeno, sin observar diferencias entre los machos castrados y los no castrados. Esta diferencia fue más acentuada en las edades intermedias (edad reproductiva). En las muestras pulmonares se observó un incremento de neutrófilos y monocitosmacrófagos inflamatorios relacionado con el atraso en la señalización de IFN tipo I en los pulmones de machos y hembras ovariectomizadas, aumento de citocinas y quimiocinas, lesión vascular y alteración en la respuesta de los linfocitos $\mathrm{T}$ y, por otro lado, mayor tasa de replicación viral en los epitelios respiratorios de los machos. Estos hallazgos demuestran un papel protector derivado de la señalización estrogénica con su receptor, el cual es notorio durante la edad reproductiva en el sexo femenino ${ }^{(50)}$.

Aunque se ha encontrado la presencia de receptores de la ECA-2 en espermatogonias que expresan genes asociados a una mayor reproducción y transmisión viral y en las células de Sertoli y Leydig humanas ${ }^{(44)}$, no se ha logrado confirmar claramente el compromiso gonadal causado por el SARS-CoV-2 en humanos, a diferencia de otros coronavirus, como el SARS$\mathrm{CoV}$, que han causado daño testicular y defectos en la espermatogénesis dada por amplia destrucción de células germinales, pobre cantidad de espermatozoides en los túbulos seminíferos y presencia de importante infiltrado inflamatorio ${ }^{(51)}$. En un pequeño estudio realizado por el grupo de la Universidad Médica de Nanjing, en China, en 13 pacientes, 12 de ellos que se encontraban entre la segunda y la tercera década de vida, durante la fase aguda y de recuperación por COVID-19 y 1 de 67 años, que falleció, no se pudo demostrar la presencia del ARN del coronavirus en las muestras seminales ni del espécimen testicular del paciente fallecido ${ }^{(52)}$. Otro estudio retrospectivo, llevado a cabo en Wuhan, en 81 pacientes con COVID-19 entre los 20 y los 54 años, de los cuales, 70 desarrollaron enfermedad moderada, 7 desarrollaron enfermedad grave y 2 fueron críticos, comparados con 100 controles saludables con recientes evaluación de su función gonadal, mostró una elevación significativa de los niveles de prolactina y de HL, y la relación testosterona/HL y hormona foliculoestimulante (FSH)/HL estuvo disminuida de manera significativa. La elevación de la prolactina puede ser multifactorial, pero el hecho de hallarse un aumento de la HL asociado con una disminución de la relación testosterona/HL sugiere daño en las células de Leydig. Asimismo, en los casos graves se observó una mayor elevación de la proteína C-reactiva, que, acompañada por la liberación de citocinas inflamatorias, como el IFN, puede comprometer la función testicular ${ }^{(53)}$. 


\section{Áreas inciertas}

- ¿Existe un peor pronóstico en hombres con hipogonadismo y en terapia de reemplazo hormonal con testosterona? Este interrogante surge a partir de observaciones en las que la testosterona inhibe el estímulo inmunitario inducido por la secreción de citocinas proinflamatorias, como el interferón gamma (IFN- $\gamma$ ) y el factor de necrosis tumoral (FNT). Por otro lado, se ha observado un mayor riesgo de trombosis venosa profunda, de acuerdo con estudios previos en hombres con hipogonadismo y en terapia de reemplazo ${ }^{(54,55)}$.

- ¿Qué efectos a largo plazo se pueden presentar sobre la función gonadal en hombres que desarrollaron COVID-19?

- ¿La alopecia androgénica, que es dependiente de las variantes halladas en el gen del receptor de andrógenos, localizado en el cromosoma $\mathrm{X}$, puede ser una condición de riesgo de mayor gravedad para COVID-19? Esto, de acuerdo con un estudio preliminar observacional realizado en España, en 41 pacientes hospitalizados por neumonía bilateral por COVID-19 y que exhibían esta condición, aunque con varios sesgos (de observador, escala subjetiva, entre otros), que podría servir de marcador para estudios futuros ${ }^{(56)}$.

- ¿Existe un peor pronóstico en otras patologías o condiciones relacionadas con la anovulación crónica, según los hallazgos observados en modelos murinos, o depende de las condiciones de autorregulación de los receptores estrogénicos?

\section{Conclusiones}

El comportamiento de la enfermedad por SARS-CoV-19 varía de acuerdo con el sexo del individuo según diversos factores relacionados con la inmunidad celular y humoral regulada por las hormonas sexuales y su expresión gonadal; sin embargo, aún es precoz definir la presencia de secuelas reproductivas para establecer las estrategias preventivas o de manejo. Es claro que el papel que desempeñan los estrógenos y su efecto inmunoprotector abre las expectativas de ampliar las líneas de investigación para dirigir las terapias encaminadas a la regulación de la respuesta inflamatoria, que, en últimas, lleva a consecuencias deletéreas graves en los individuos afectados. Asimismo, queda por explorar y definir si existe la posibilidad de transmisión vertical y, de ser así, qué factores se aplicarían a esta.

\section{Conflictos de interés}

Los autores declaran no tener ningún conflicto de interés.

\section{Financiación}

Los autores reportan que no recibieron financiación para la realización del presente documento.

\section{Referencias}

1. Worldometer. COVID-19 Coronavirus Pandemic [Internet]. Disponible en: https://www.worldometers.info/coronavirus/.

2. Suba Z. Prevention and Therapy of COVID-19 via Exogenous Estrogen Treatment for Both Male and Female Patients. J Pharm Pharm Sci. 2020;23(1):75-85. doi: 10.18433/jpps31069.

3. Coronaviridae Study Group of the International Committee on Taxonomy of Viruses. The species Severe acute respiratory syndrome-related coronavirus: classifying 2019-nCoV and naming it SARS-CoV-2. Nat Microbiol. 2020;5(4):536-44. doi: 10.1038/s41564-020-0695-z.

4. Zhu N, Zhang D, Wang W, Li X, Yang B, Song J, et al. A novel coronavirus from patients with pneumonia in China, 2019. N Engl J Med. 2020;382(8):72733. doi: 10.1056/NEJMoa2001017.

5. Lu R, Zhao X, Li J, Niu P, Yang B, Wu H, et al. Genomic characterisation and epidemiology of 2019 novel coronavirus: implications for virus origins and receptor binding. Lancet. 2020;395(10224):565-74. doi: 10.1016/S01406736(20)30251-8.

6. Li Q, Guan X, Wu P, Wang X, Zhou L, Tong L, et al. Early transmission dynamics in Wuhan, China, of novel coronavirus-infected pneumonia. $\mathrm{N}$ Engl J Med. 2020;382(13):1199-207. doi: 10.1056/NEJMoa2001316.

7. Paraskevis D, Kostaki EG, Magiorkinis G, Panayiotakopoulos G, Sourvinos $\mathrm{G}$, Tsiodras S. Full-genome evolutionary analysis of the novel corona virus (2019-nCoV) rejects the hypothesis of emergence as a result of a recent recombination event. Infect Genet Evol. 2020;79:104212. doi: 10.1016/j. meegid.2020.104212.

8. Tang X, Wu C, Li X, Song Y, Yao X, Wu X, et al. On the origin and continuing evolution of SARS-CoV-2. Nat Sci Rev. 2020;0:1-2. doi: 10.1093/nsr/ nwaa036.
9. Epidemiology Working Group for NCIP Epidemic Response; Chinese Center for Disease Control and Prevention. [The epidemiological characteristics of an outbreak of 2019 novel coronavirus diseases (COVID-19) in China]. Zhonghua Liu Xing Bing Xue Za Zhi. 2020;41(2):145-51. doi: 10.3760/cma. j.issn.0254-6450.2020.02.003.

10. Grasselli G, Zangrillo A, Zanella A, Antonelli M, Cabrini L, Castelli A, et al. Baseline characteristics and outcomes of 1591 patients infected with SARS-CoV-2 admitted to ICUs of the Lombardy Region, Italy. JAMA. 2020;323(16):1574-81. doi: https://doi.org/10.1001/jama.2020.5394.

11. Gov.co. Datos Abiertos. Estado de Casos de Coronavirus COVID-19 en Colombia [Internet]. Disponible en: https://bit.ly/36JqVrZ.

12. The Novel Coronavirus Pneumonia Emergency Response Epidemiology Team. Vital Surveillances: The Epidemiological Characteristics of an Outbreak of 2019 Novel Coronavirus Diseases (COVID-19). China, 2020. China CDC Weekly. 2020;2(8):113-22.

13. Kadel S, Kovats S. Sex Hormones Regulate Innate Immune Cells and Promote Sex Differences in Respiratory Virus Infection. Front Immunol 2018;9:1653. doi: 10.3389/fimmu.2018.01653.

14. Chan JF, Yuan S, Kok KH, To KKW, Chu H, Yang J, et al. A familial cluster of pneumonia associated with the 2019 novel coronavirus indicating person-to-person transmission: a study of a family cluster. Lancet. 2020;395(10223):514-23. doi: 10.1016/S0140-6736(20)30154-9.

15. Zhang W, Du RH, Li B, Zheng XS, Yang XL, Hu B, et al. Molecular and serological investigation of 2019-nCoV infected patients: implication of multiple shedding routes. Emerg Microbes Infect. 2020;9(1):386-9. doi: $10.1080 / 22221751.2020 .1729071$. 
16. To KK, Tsang OT, Chik-Yan Yip C, Chan KH, Wu TC, et al. Consistent detection of 2019 novel coronavirus in saliva. Clin Infect Dis. 2020;ciaa149. doi: $10.1093 / \mathrm{cid} / \mathrm{ciaa} 149$.

17. Xia J, Tong J, Liu M, Shen Y, Guo D. Evaluation of coronavirus in tears and conjunctival secretions of patients with SARS-CoV-2 infection. J Med Virol. 2020;10.1002/jmv.25725. doi: 10.1002/jmv.25725.

18. Young K. COVID-19: Virus in Semen/DVT Findings on Autopsy/Anakinra [Internet]. NEJM Journal Watch. 2020. Disponible en: https://bit.ly/2UfFxdx.

19. Li D, Jin M, Bao, P, Zhao W, Zhang S. Clinical Characteristics and Results of Semen Tests Among Men With Coronavirus Disease 2019. JAMA Netw Open. 2020;3(5):e208292. doi: 10.1001/jamanetworkopen.2020.8292.

20. Lauer SA, Grantz KH, Bi Q, Jones FK, Zheng Q, Meredith HR, et al. The incubation period of coronavirus disease 2019 (COVID-19) from publicly reported confirmed cases: estimation and application. Ann Intern Med. 2020;172(9):57782. doi: 10.7326/M20-0504.

21. Jiang X, Niu Y, Li X, Li L, Cai W, Chen Y, et al. Is a 14-day quarantine period optimal for effectively controlling coronavirus disease 2019 (COVID-19)? medRxiv. 2020. doi: https://doi.org/10.1101/2020.03.15.20036533.

22. Yu P, Zhu J, Zhang Z, Han Y. A familial cluster of infection associated with the 2019 novel coronavirus indicating potential person-to-person transmission during the incubation period. J Infect Dis. 2020;221(11):1757-61. doi: 10.1093/ infdis/jiaa077.

23. Du Z, Xu X, Wu Y, Wang L, Cowling BJ, Meyers LA, et al. Serial interval of COVID-19 among publicly reported confirmed cases. Emerg Infect Dis. 2020;26(6)1341-3. doi: 10.3201/eid2606.200357.

24. Chen H, Guo J, Wang C, Luo F, Yu X, Zhang W, et al. Clinical characteristics and intrauterine vertical transmission potential of COVID-19 infection in nine pregnant women: a retrospective review of medical records. Lancet. 2020;395(10226):809-15. doi: 10.1016/S0140-6736(20)30360-3.

25. Schwartz DA, Graham AL. Potential maternal and infant outcomes from (Wuhan) Coronavirus 2019-nCoV infecting pregnant women: Lessons from SARS, MERS, and other human coronavirus infections. Viruses. 2020;12(2):194. doi: $10.3390 / \mathrm{v} 12020194$.

26. Karimi-Zarchi M, Neamatzadeh H, Dastgheib SA, Abbasi H, Mirjalili SR, Behforouz A, et al. Vertical transmission of coronavirus disease 19 (COVID-19) from infected pregnant mothers to neonates: a review. Fetal Pediatr Pathol. 2020;1-5. doi: 10.1080/15513815.2020.1747120

27. Schwartz DA. An analysis of 38 pregnant women with COVID-19, their newborn infants, and maternal- fetal transmission of SARS-CoV-2: maternal coronavirus infections and pregnancy outcomes. Arch Pathol Lab Med. 2020. doi: 10.5858/arpa.2020-0901-SA.

28. Wang S, Guo L, Chen L, Liu W, Cao Y, Zhang J, et al. A case report of neonatal COVID-19 infection in China. Clin Infect Dis. 2020; ciaa225. doi: 10.1093/cid/ ciaa225

29. Zhu H, Wang L, Fang C, Peng S, Zhang L, Chang G, et al. Clinical analysis of 10 neonates born to mothers with 2019-nCoV pneumonia. Transl Pediatr. 2020;9(1):51-60. doi: 10.21037/tp.2020.02.06.

30. Zeng H, Xu C, Fan J, Tang Y, Deng Q, Zhang W, et al. Antibodies in infants born to mothers with COVID-19 pneumonia. JAMA. 2020;323(18):1848-9. doi: 10.1001/jama.2020.4861.

31. Dong L, Tian J, He S, Zhu C, Wanh J, Liu C, et al. Possible vertical transmission of SARS-CoV-2 from an infected mother to her newborn. JAMA. 2020;323(18):1846-8. doi: 10.1001/jama.2020.4621

32. Favre G, Pomar L, Musso D, Baud D. Epidemia 2019-nCoV: ¿qué pasa con los embarazos? Lancet. 2020;395(10224):e40. doi: 10.1016/S0140-6736.

33. Wong SF, Chow KM, Leung TN, Ng WF, Ng Tk, Sjek CC, et al. Pregnancy and Perinatal Outcomes of Women With Severe Acute Respiratory Syndrome. Am J Obstet Gynecol. 2004;191(1):292-7. doi: 10.1016/j.ajog.2003.11.019.

34. Vivanti A, Vauloup-Fellous C, Prevot S, Zupan V, Suffee C, Do-Cao J, et al. Transplacental transmission of SARS-CoV-2 infection [Internet]. Nature Research. 2020. doi: 10.21203/rs.3.rs-28884/v1.

35. Chen L, Li Q, Zheng D, Jiang H, Wei Y, Zou L, et al. Clinical Characteristics of Pregnant Women with Covid-19 in Wuhan, China. N Engl J Med. 202;NEJMc2009226. doi: 10.1056/NEJMc2009226.

36. Guan W, Ni Z, Hu Y, Liang WH, Ou CQ, He JX, et al. Clinical characteristics of coronavirus disease 2019 in China. N Engl J Med. 2020;382(18):1708-20. doi: 10.1056/NEJMoa2002032.
37. Chen N, Zhou M, Dong X, Qu J, Gong F, Han Y, et al. Epidemiological and clinical characteristics of 99 cases of 2019 novel coronavirus pneumonia in Wuhan, China: a descriptive study. Lancet. 2020;395(10223):507-13. doi: 10.1016/S0140-6736(20)30211-7.

38. Sutton D, Fuchs K, D'Alton M, Goffman D. Universal Screening for SARS CoV-2 in Women Admitted for Delivery. N Engl J Med. 2020;382(22):21634. doi: 10.1056/NEJMc2009316.

39. Peña-López BO, Rincón-Orozco B. Generalidades de la pandemia po COVID-19 y su asociación genética con el virus del SARS. Salud UIS. 2020;52(2):83-6. doi: http://dx.doi.org/10.18273/revsal.v52n2-2020001.

40. Hoffmann M, Kleine-Weber H, Schroeder S, Kruger N, Herrler T, Erichsen S, et al. SARS-CoV-2 cell entry depends on ACE2 and TMPRSS2 and is blocked by a clinically proven protease inhibitor. Cell. 2020;181(2):271-280.e8. doi: 10.1016/j.cell.2020.02.052.

41. Fernández-Serra A, Rubio-Briones B, García-Casado Z, Solsona E, LópezGuerrero JA. Cáncer de próstata: la revolución de los genes de fusión. Actas Urol Esp. 2011;35(7):420-8.

42. Wambier CA, Goren A. Severe acute respiratory syndrome coronavirus (SARS-CoV-2) infection is likely to be androgen mediated. I Am Acad Dermatol. 2020;S0190-9622(20)30608-3. doi: https://doi.org/10.1016/j. jaad.2020.04.032.

43. Sharifi N, Ryan CJ. Androgen hazards with COVID-19. Endocr Relat Cancer 2020;27(6):E1-E3. doi: 10.1530/ERC-20-0133.

44. Wang Z, Xu X. scRNA-seq Profiling of Human Testes Reveals the Presence of the ACE2 Receptor, A Target for SARS-CoV-2 Infection in Spermatogonia, Leydig and Sertoli Cells. Cells. 2020;9(4):920. doi:10.3390/cells9040920.

45. Gheblawi M, Wang K, Viveiros A, Nguyen Q, Zhong J, Turner AJ, et al. Angiotensin-Converting Enzyme 2: SARS-CoV-2 Receptor and Regulator of the Renin-Angiotensin System. Circ Res. 2020;126(10):1457-75. doi: 10.1161/ CIRCRESAHA.120.317015.

46. Douglas GC, O'Bryan MK, Hedger MP, Lee DK, Yarski MA, Smith AI, et al. The novel angiotensin-converting enzyme (ACE) homolog, ACE2, is selectively expressed by adult Leydig cells of the testis. Endocrinology. 2004;145(10):4703-11. doi: 10.1210/en.2004-0443.

47. Shaw AC, Goldstein DR, Montgomery RR. Age-dependent dysregulation of innate immunity. Nat Rev Immunol. 2013;13(12):875-87. doi: 10.1038/ nri3547.

48. Mesa-Villanueva M, Patiño PJ. Receptores tipo Toll: entre el reconocimiento de lo no propio infeccioso y las señales endógenas de peligro. Inmunología. 2006;25(2):115-30.

49. Kovats S. Estrogen receptors regulate innate immune cells and sig naling pathways. Cell Immunol. 2015;294(2):63-9. doi: 10.1016/j.cellimm.2015.01.018.

50. Channappanavar R, Fett C, Mack M, Ten Eyck PP, Meyerholz DK, Perlman S. Sex-based differences in susceptibility to severe acute respiratory syndrome coronavirus infection. J Immunol. 2017;198(10):4046-53. doi: 10.4049/jimmunol.1601896.

51. Xu J, Qi L, Chi X, Yang J, Wei X, Gong E, et al. Orchitis: A Complication of Severe Acute Respiratory Syndrome (SARS). Biol Reprod. 2006;74(2):410-6. doi: 10.1095/biolreprod.105.044776.

52. Song C, Wang Y, Li W, Hu B, Chen G, Xia P, et al. Absence of 2019 novel coronavirus in semen and testes of COVID-19 patients. Biol Reprod. 2020;ioaa050. doi: 10.1093/biolre/ioaa050.

53. Ma L, Xie W, Li D, Shi L, Mao Y, Xiong Y, et al. Effect of SARS-CoV-2 infection upon male gonadal function: A single center-based study. medRxiv. 2020. doi: https://doi.org/10.1101/2020.03.21.20037267.

54. Walker RF, Zakai NA, MacLehose RF, Cowan LT, Adam TJ, Alonso A, et al. Association of testosterone therapy with risk of venous thromboembolism among men with and without hypogonadism. JAMA Intern Med. 2019;180(2):190-7. doi: 10.1001/jamainternmed.2019.5135.

55. La Vignera S, Cannarella R, Condorelli RA, Torre F, Aversa A Calogero A Sex-Specific SARS-CoV-2 Mortality: Among Hormone-Modulated ACE2 Expression, Risk of Venous Thromboembolism and Hypovitaminosis D. Int J Mol Sci. 2020;21(8):2948. doi:10.3390/ijms21082948.

56. Goren A, Vaño-Galván S, Wambier CG, McCoy J, Gomez-Zubiaur A, MorenoArrones OM, et al. A preliminary observation: male pattern hair loss among hospitalized CoVid-19 patients in Spain - a potential clue to the role of androgens in covid-19 severity. J Cosmet Dermatol. 2020. doi: 10.1111/ jocd.13443. 